Pacific Journal of Mathematic 


\title{
A NOTE ON CONTINUOUS COLLECTIONS OF DISJOINT CONTINUA
}

\author{
E. I. Bethel
}

M. E. Hamstrom has shown that if $G$ is a continuous collection of disjoint ares filling up a compact continuous curve $M$ in the plane such that $M / G$ is an arc, then $G^{*}\left(x \in G^{*}\right.$ if and only if for some $g \in G, x \in g$ ) is a simple closed curve plus its interior. One purpose of this note is to show that if $S$ is a space satisfying Axioms $0-5$ of R. L. Moore's Foundations of Point Set Theory, and $M \subset S$ such that (1) $M$ has one and only one complementary domain, and (2) there exists a continuous collection of disjoint nondegenerate continua filling up $M$, then $M$ is a simple closed curve $J$ plus one of the complementary domains of $J$. Another purpose of this note is to state and prove some consequences of this theorem.

Both circle-like continuum and an annulus-like point set are defined in a natural way. It is shown that if $M$ is a compact continuum in a separable space satisfying Axioms $0-5$ of $R$. L. Moore, such that (1) $M$ is filled up by a continuous collection of disjoint continua, (2) all but countably many elements of $G$ are circlelike, and (3) there exists a point 0 not in $M$ such that no element of $G$ separates two elements of $G$ from one another and both from 0 in $S$, then $M / G$ is an arc. An example exists where the conclusion of the theorem fails if condition (3) is omitted, but it is not included in this note. From the preceding theorem, it follows that if $M$ is a compact continuum in the plane which is filled up by a continuous collection of disjoint circle-like continua, then $M$ is annulus-like.

Throughout this note $\operatorname{Bd}(M)$ will denote the boundary of $M$ and $\mathrm{Cl}(M)$ will denote the closure of $M$. Furthermore, $S$ will denote a space satisfying Axioms $0-5$ of [3] unless otherwise stated, and "simple closed curve" will be abbreviated scc.

Definition 1. The collection $G$ is a continuous collection if and only if $\left\{g_{n}\right\}$ is a sequence of elements of $G$ such that for some $g \in G$, $\lim \inf \left\{g_{n}\right\} \cap g \neq \varnothing$, then lim inf $\left\{g_{n}\right\}=\lim \sup \left\{g_{n}\right\}=g$ (We shall denote this by $\left\{g_{n}\right\} \rightarrow g$ ).

Lemma 1. Let $M$ denote a point set and let $N$ denote a proper subset of $M$ such that $M-N$ is connected. Let $J \subset N$ such that $M-J$ is the union of two mutually separated point sets $H$ and $K$ ( $H$ and $K$ are disjoint and neither contains a limit point of the other). Then one of $H$ and $K$ is a subset of $N$. 
Proof. Let $D=M-N$. Since $D$ is connected $D \subset H$ or $D \subset K$, say $K$. Now suppose $H \not \subset N$. Then $H \cap D \neq \varnothing$. This means that $H \cap K \neq \varnothing$, a contradiction.

Lemma 2. Suppose $M$ is a closed subset of $S$ such that $M \neq S$ and $S-M$ is connected and every component of $S-\mathrm{Cl}(D)$ is the interior of a scc, where $D=S-M$ and the ideal point is a point of $D$. Then if $X$ and $Y$ are components of $S-\mathrm{Cl}(D)$ such that $\mathrm{Cl}(X) \cap \mathrm{Cl}(Y) \neq \varnothing$, then $\mathrm{Cl}(X) \cap \mathrm{Cl}(Y)$ is degenerate.

Proof. Let us suppose the contrary. Let $P$ and $O$ denote points of $\operatorname{Bd}(X) \cap \operatorname{Bd}(Y)$. Both $P$ and $O$ are accessible from both $X$ and $Y$ (Theorem 59, Chapt. IV of [3]). There exist ares $(O A P)$ and $(O B P)$ such that $[(O A P)-(A \cup P)] \subset X$ and $[(O B P)-(O \cup P)] \subset Y$. Now the sce $J=[(O B P) \cup(O A P)] \subset M$. It follows from Lemma 1 that the interior $I$ of $J$ is a subset of $M$. Since $B \in Y, I \cap Y \neq \varnothing$. Likewise, $I \cap X \neq \varnothing$. But $X \cup I$ is a connected subset of $S-\operatorname{Cl}(D)$ of which $X$ is a proper subset contrary to the fact that $X$ is a component of $S-\mathrm{Cl}(D)$. Hence $\mathrm{Cl}(X) \cap \mathrm{Cl}(Y)$ is degenerate.

LEMMA 3. If $G$ is a continuous collection of disjoint closed point sets such that each element of $G$ is a limit element of $G$, then no element of $G$ contains a domain.

Proof. Let $g \in G$ and $D=G^{*}-g$. Since each element of $G$ is a limit element of $G$, some point of $G$ is a limit point of $D$. Since $g$ is closed, there exists a sequence $\left\{g_{n}\right\}$ of elements of $G$ such that for each $n, g_{n} \subset D$ and $\left\{g_{n}\right\} \rightarrow g$. If $g$ contains a domain $U$ this would imply that for some $k, g_{k} \cap U \neq \varnothing$ since $G$ is a continuous collection, contrary to the fact that the elements of $G$ are disjoint.

THeOREM 1. If $M$ is a compact continuous curve in $S$ such that $M \neq S$ and does not separate $S$, and $M$ is filled up by a continuous collection $G$ of disjoint continua not all degenerate, then $M$ contains a domain.

Proof. Let $g \in G$ such that $g$ is nondegenerate, and let $P_{1}$ and $P_{2}$ denote points of $g$. Let $T$ denote a component of $M-g$. Since $M$ is compact and $G$ is a continuous collection, $g=\mathrm{Bd}(T)$ with respect to $M$. Since $M$ is a continuous curve, there exist open connected subsets $R_{1}$ and $R_{2}$ of $M$ such that $P_{1} \in R_{1}, P_{2} \in R_{2}$, and $\mathrm{Cl}\left(R_{1}\right) \cap \mathrm{Cl}\left(R_{2}\right)=$ $\varnothing$. In the following $i=1,2$. Let $0_{i} \in R_{i} \cap T$. Now there exist arcs $0_{i} P_{i} \subset R_{i}$. Let $A_{i}$ denote the first point in the order from $0_{i}$ to $P_{i}$ of $\left(0_{i} P_{i}\right) \cap g$. Now $\left[\left(0_{i} A_{i}\right)-A_{i}\right] \subset T$. Since $T$ is a connected open subset 
of $M$, there is an arc $\left(0_{1} 0_{2}\right) \subset T$ (Theorem 13, Chapt. II of [3]). Let $U=\left(0_{1} 0_{2}\right) \cup\left(0_{1} A_{1}\right) \cup\left(0_{2} A_{2}\right)$. Now $U$ is a continuous curve. Hence, there is an $\operatorname{arc}\left(A_{1} A_{2}\right) \subset U$. Now $\left[\left(A_{1} A_{2}\right)-\left(A_{1} \cup A_{2}\right)\right] \subset T$, and $\left(A_{1} A_{2}\right) \cup g$ separates $S$ (Theorem 22, Chapt. IV of [3]). Let $H$ denote one of the complementary domains of $\left(A_{1} A_{2}\right) \cup g$ and let $K$ denote all other complementary domains of $\left(A_{1} A_{2}\right) \cup g$. According to Lemma 1 , one of the point sets $H$ and $K$ is a subset of $M$. This means that $M$ contains a domain.

THEOREM 2. Under the hypothesis of Theorem 1, it follows that each component of $M-\operatorname{Bd}(D)$ is bounded by a scc, where $D=S-M$.

Proof. Let $E$ denote a complementary domain of $S-\mathrm{Cl}(D)$. By Theorem $1, E \neq \varnothing$. Let $J$ denote the outer boundary of $D$ with respect to $E$ (the outer boundary of $D$ with respect to $E$ is the boundary of the component of $S-\mathrm{Cl}(D)$ containing $E)$. Now $\operatorname{Bd}(D)$ is a continuous curve (Theorem 42, Chapt. IV of [3]). Furthermore, $J$ is a scc (Theorem 43, Chapt. IV of [3]). Since $E$ is a component of $S-\mathrm{Cl}(D), \operatorname{Bd}(E)=J$.

Theorem 3. Under the hypothesis of Theorem 1 with the additional stipulation that no element of $G$ is degenerate, it follows that $\operatorname{Bd}(M)$ is a scc.

Proof. Let $C$ denote a complementary domain of $S-\mathrm{Cl}(D)$, where $D=S-M$. By Theorem $2, \operatorname{Bd}(C)$ is a scc. Let $g \in G$. It follows that if $g \cap(C \cup \mathrm{Bd}(C)) \neq \varnothing, g \subset(C \cup \mathrm{Bd}(C))$. Let us prove this by contradiction. Let $P \in g-(C \cup \mathrm{Bd}(C))$. Now $g \cap \mathrm{Bd}(C) \neq \varnothing$ since $g$ is a continuum. There is a subcontinuum $g^{\prime}$ of $g$ which is irreducible from $P$ to $\operatorname{Bd}(C)$. Let 0 denote a point of $C$ and let $R$ denote an open connected subset of $M$ such that $P \in R$ and $\mathrm{Cl}(R) \cap \mathrm{Bd}(C)=\varnothing$. $\quad \mathrm{By}$ Lemma 3, $C \not \subset g$. Furthermore, $\mathrm{Bd}(C) \not \subset g$. Suppose this is the case. Now

$$
H=(C \cup \operatorname{Bd}(C))-[(C \cup \operatorname{Bd}(C)) \cap g]=\varnothing .
$$

Furthermore, $(C \cup \operatorname{Bd}(C)) \cap g$ must contain a limit point of $H$. If $t \in G$ and $H \cap t \neq \varnothing$, then $t \in C$. But there exists a sequence $\left\{g_{n}\right\}$ of elements of $G$ such that $\left\{g_{n}\right\} \rightarrow g$. This means that there is a $g_{k}$ of $\left\{g_{n}\right\}$ such that $g_{k} \cap \mathrm{Bd}(C) \neq \varnothing$, contrary to the fact that the elements of $G$ are disjoint. Hence, $\operatorname{Bd}(C)-(g \cap \operatorname{Bd}(C)) \neq \varnothing$. Now $C$ lies in some component $E$ of $M-g^{\prime}$. Furthermore, each component of $[\operatorname{Bd}(C)-(g \cap \operatorname{Bd}(C))] \subset E$ since each point of $\operatorname{Bd}(C)-(g \cap \operatorname{Bd}(C))$ is a limit point of $C$. Let $K$ denote a component of $\operatorname{Bd}(C)-(g \cap \operatorname{Bd}(C))$. 
There exists a sequence of elements $\left\{g_{n}\right\}$ of $G$ such that $\left\{g_{n}\right\} \rightarrow g$ and for each $n, g_{n} \cap R \neq \varnothing$. This in turn means that $P$ is a limit point of $E$. Since $P$ is a limit point of $E, R$ contains some point $X$ of $E$. There is an arc $(X P) \subset R$. Let $A$ denote the first point in the order from $X$ to $P$ of $(X P) \cap g^{\prime}$. Since $E$ is connected open subset of $M$, there is an arc $(X 0) \subset E$. Let $B$ denote the first point in the order from $X$ to 0 of $(X 0) \cap \operatorname{Bd}(C)$. Now there exists an arc $(A B)$ such that $(A B) \subset((X A) \cup(X B))$. Let $U$ denote a point of $g^{\prime} \cap \operatorname{Bd}(C)$. There is an arc $(A U)$ such that $[(A U)-(A \cup U)] \subset C$. Let $Z$ denote a point of the $\operatorname{arc}(A B)$ such that $Z \neq A$ and $Z \neq B$. Then we have two disjoint compact continua $H$ and $g^{\prime}$ and two ares $(Z A)$ and $(B U)$ such that $H \cap(Z A)=$ $A, H \cap(B U)=B,(Z A) \cap g^{\prime}=A$ and $(B U) \cap g^{\prime}=U$. Let $D_{H}$ denote the component of $S-g^{\prime}$ such that $H \subset D_{H}$, and let $D_{g}$, denote the component of $S-H$ such that $g^{\prime} \subset D_{g^{\prime}}$. Moreover, let $H^{\prime}=S-D_{g^{\prime}}$, $K^{\prime}=S-D_{H}$ and let $N=H^{\prime} \cup K^{\prime} \cup(Z A) \cup(B U) . \quad S-N$ is the union of two disjoint connected domains $D_{1}$ and $D_{2}$ such that $(Z A)$ and $(B U)$ is a subset of both $\operatorname{Bd}\left(D_{1}\right)$ and $\operatorname{Bd}\left(D_{2}\right)$ (Theorem 38, Chapt. IV of [3]). It follows from Lemma 1 that either $D_{1} \subset M$ or $D_{2} \subset M$, say $D_{1}$. Since $[(Z A) \cup(B U)] \subset \operatorname{Bd}\left(D_{1}\right)$, it follows that $D_{1} \cap C \neq \varnothing$ and $D_{1} \cap(S-C) \neq \varnothing$. Hence, $C$ is a proper subset of $D_{1}$ which means that $C$ is not a component of $S-\mathrm{Cl}(D)$, a contradiction. Hence, $g \subset(C \cup \mathrm{Bd}(C))$. By a similar argument, it may be shown that if $g \in G$, there is some component $C$ of $S-\operatorname{Cl}(D)$ such that $g \cap \operatorname{Cl}(C) \neq \varnothing$.

Now let us suppose that $S-\mathrm{Cl}(D)$ has more than one component. Let $W$ denote a set such that $X \in W$ if and only if for some component $C$ of $S-\mathrm{Cl}(D), X=C \cup \operatorname{Bd}(C)$. Now $W^{*}=M$. This follows from the immediately preceding paragraph. For some two point sets $X$ and $Y$ of $W, X \cap Y \neq \varnothing$. Otherwise, $M$ would be the union of a countable number of closed and disjoint point sets. Let $P$ denote a point of $\operatorname{Bd}(X) \cap \operatorname{Bd}(Y)$, and let $g \in G$ such that $P \in g$. Since $g \cap X \neq \varnothing, g \subset X$. Likewise, $g \subset Y$. By Lemma $2, X \cap Y$ is degenerate, a contradiction. Hence, $W=X$. But $\operatorname{Bd}(X)$ is a scc. Hence, $M$ is bounded by a sce.

CoROLlary. A necessary and sufficient condition that a compact continuous curve $M$ which does not separate the plane be a simple closed curve plus its interior is that there exist a continuous collection of nondegenerate disjoint continua filling up $M$.

Proof. The sufficiency follows from Theorem 3. The necessity follows from the fact that each scc plus its interior in the plane is homeomorphic to the unit square plus its interior, and there is a continuous collection of disjoint nondegenerate continua filling up the unit square. This collection is invariant under a homeomorphism. 
Theorem 4. Let $M$ denote a compact continuous curve in $S$ such that $M \neq S$ and $M$ is filled up by a continuous collection $G$ of disjoint nondegenerate continua. Then the boundary of every complementary domain of $M$ is a scc.

Proof. If $M$ has only one complementary domain, the theorem follows from Theorem 3. Let $D$ denote a complementary domain of $M$ and let $H$ denote the collection of all other complementary domains of $M$. Let $L=M \cup H^{*}$ and let $C$ denote a component of $S-\mathrm{Cl}(D)$. It follows as in Theorem $2, \operatorname{Bd}(C)$ is a simple closed curve. Let $g \in G$ such that $g \cap \operatorname{Cl}(C) \neq \varnothing$. Then $g \subset \mathrm{Cl}(C)$. Suppose the contrary. Let $P \in(g-g \cap \mathrm{Bd}(C))$. Now suppose $\mathrm{Bd}(C) \subset g$. There is some component $V$ of $M-g$ which is not a subset of $C$. For if all components of $M-g$ lie in $C$, then $G$ is not a continuous collection. Now $g$ is $\operatorname{Bd}(V)$ with respect to $M$. A process like that used in Theorem 3 is used to construct two $\operatorname{arcs}(A X B)$ and $(A Y B)$ such that $A$ and $B$ are points of $\mathrm{Bd}(C),[(A X B)-(A \cup B)] \subset V$, and $[(A Y B)-(A \cup B)] \subset C$. Then by Lemma 1 , one of the complementary domains of the sec $(A X B) \cup(A Y B) \subset L$. This means that $C$ is not a component of $S-$ $\mathrm{Cl}(D)$, a contradiction. Hence, $\mathrm{Bd}(C) \not \subset g$. Let $g^{\prime}$ denote a subcontinuum of $g$ which is irreducible from $P$ to $g \cap \operatorname{Bd}(C)$. Now every component of $\mathrm{Bd}(C)-(g \cap \mathrm{Bd}(C))$ has a limit point in $g \cap \operatorname{Bd}(C)$. Now $C \cup(\operatorname{Bd}(C)-$ $g \cap \mathrm{Bd}(C)$ ) lies in some component $U$ of $L-g^{\prime}$. Let $K$ denote a component of $\operatorname{Bd}(C)-g \cap \operatorname{Bd}(C)$. There exists a sequence of elements $\left\{g_{n}\right\}$ of $G$ such that $\left\{g_{n}\right\} \rightarrow g$ and $g_{n} \cap K \neq \varnothing$ for each $n$. This means that $P$ is a limit point of $U$. Let $Z$ denote a point of $g^{\prime} \cap \operatorname{Bd}(C)$. As in Theorem 3, we construct arcs as there and again apply Theorem 36 of Chapt. IV of [3] and reach the contradiction that $C$ is not a component of $S-\mathrm{Cl}(D)$. Hence, as in Theorem 3, if $g \in G$ such that $g \cap \mathrm{Cl}(C) \neq \varnothing, g \subset \mathrm{Cl}(C)$. Likewise, it easily follows that if $g \in G$, then for some $C$ of $S-\mathrm{Cl}(C), g \cap \mathrm{Cl}(C) \neq \varnothing$. Again we may define the point set $W$ as in Theorem 3 and show that it contains only one point set using the fact that no compact continuous curve has uncountably many complementary domains (Theorem 63, Chapt. IV of [3]), i.e., the point set $W$ again is a countable collection. It then follows that $L$ is a simple closed curve plus one of its complementary domains. Consequently, $D$ is bounded by a sce.

COROLlary 1. Let $G$ be a continuous collection of nondegenerate disjoint continua filling up $S$. Then there is no nondegenerate subcollection $G^{\prime}$ (contains at least two elements of $G$ ) of $G$ such that $\left(G^{\prime}\right)^{*}$ is a continuous curve.

Proof. Suppose the contrary. Since $\left(G^{\prime}\right)^{*}$ satisfies the hypothesis 
of Theorem 4, each complementary domain of $\left(G^{\prime}\right)^{*}$ is bounded by a simple closed curve. Let $D$ denote a complementary domain of $\left(G^{\prime}\right)^{*}$, and let $g \in G$ such that $P \in g$. Now $g \subset \operatorname{Bd}(D)$ since $G$ is a continuous collection. This means that $\operatorname{Bd}(D)$ must be the union of a countable collection of nondegenerate disjoint continua.

R. D. Anderson has stated in [1] that there exists a continuous collection $G$ of pseudo arcs filling up a 2 -sphere. It follows from Corollary 1 that if $G^{\prime}$ is a proper nondegenerate subcollection of $G$, $\left(G^{\prime}\right)^{*}$ is not a continuous curve.

Corollary 2. Suppose $M$ is a compact continuous curve in the plane which is filled up by a continuous collection $G$ of disjoint nondegenerate continua such that $M$ has only two complementary domains such that their boundaries do not intersect. Then $M$ is an annulus.

Proof. Let $D_{1}$ and $D_{2}$ denote the complementary domains of $M$. By Theorem 4, $\operatorname{Bd}\left(D_{1}\right)$ and $\operatorname{Bd}\left(D_{2}\right)$ are scc. Now one of $\operatorname{Bd}\left(D_{1}\right)$ and $\mathrm{Bd}\left(D_{2}\right)$ separates the other from the ideal point. Otherwise, it follows that $M$ is not compact. Suppose $\operatorname{Bd}\left(D_{1}\right)$ separates $\operatorname{Bd}\left(D_{2}\right)$ from the ideal point. Now $M \subset H=I_{1}-\operatorname{Cl}\left(D_{2}\right)$, where $I_{1}$ is the interior of $\operatorname{Bd}\left(D_{1}\right)$. Furthermore, each point of $H$ belongs to some element of $G$. If not, it follows that $M$ would have more than two complementary domains. Hence, $M$ is an annulus.

Theorem 5. Let $G$ denote a continuous collection of disjoint continua is $S$ such that (1) $G$ is a compact continuous curve with respect to its elements, (2) no element of $G$ separates $S$, and (3) if $H$ is a subcollection of $G$ which is an arc with respect to its elements, then $H^{*}$ is a continuous curve and does not separate $S$. Then $G$ is either an arc or a sce with respect to its elements.

Proof. Let us suppose the contrary. Then $G$ must contain a simple triod $T$ of elements of $G$. Let $T=H_{1} \cup H_{2} \cup H_{3}$ where the $H_{i}$ are ares of elements in $G$ having a common end element. By Theorem 3, $H_{i}^{*}$ is a scc plus its interior. Let $I_{i}$ denote the interior of $\operatorname{Bd}\left(H_{i}^{*}\right)$. It easily follows that $h \subset \mathrm{Bd}\left(H_{i}^{*}\right)$. Let $A$ and $B$ denote the end points of $h$, and let $X \in h$ which is distinct from both $A$ and $B$. Let $A_{i} \in\left(\operatorname{Bd}\left(H_{i}^{*}\right)-h\right)$. Now $\left(\operatorname{Bd}\left(H_{i}^{*}\right)-h\right)=\varnothing$. Otherwise, it would follow that the elements of $G$ are not disjoint. Now the arc $\left(A A_{2} B\right)$ must lie outside $\mathrm{Bd}\left(H_{i}^{*}\right)$. Moreover, either $X$ is without $J=\operatorname{Bd}\left(H_{1}^{*}\right) \cup \operatorname{Bd}\left(H_{2}^{*}\right)$ or $A_{1}$ is without $\operatorname{Bd}\left(H_{2}^{*}\right)$. Let us suppose $X$ is without $J$. Then $A \in I_{2}$, contrary to the fact that $H_{1} \cap H_{2}=h$. Hence, $A_{1}$ is without $\operatorname{Bd}\left(H_{2}^{*}\right)$, and $(A X B)-(A \cup B)$ is a subset of the interior 
$I$ of $J$. Furthermore, $I=I_{1} \cup I_{2} \cup[(A X B)-(A \cup B)]$. Now $\left(A A_{3} B\right)-$ $(A \cup B)$ must lie outside $J$ since $\cap H_{i}=h$. Now each element of $H_{3}$ distinct from $h$ must lie outside $J$. Otherwise, $\cap H_{i} \neq h$. But $h$ is a limit element of $H_{3}$, and since $X \in h \cap I$, and $G$ is a continuous collection, $I \cap\left(H_{3}-h\right) \neq \varnothing$. Hence, $G$ does not contain a simple triod. By Theorem 75, Chapt. IV of [3], $G$ is an arc or a simple closed curve with respect to its elements.

THEOREM 6. Under the hypothesis of Theorem 5 plus the additional stipulation that one of the elements is not an end element and is not a continuous curve, it follows that $G$ must be an arc with respect to its elements.

Proof. Let us suppose $G$ is a scc with respect to its elements, and $g \in G$ is not a continuous curve. There is an arc $h k$ of elements of $G$ such that $g$ is not an end element of $h k$. Let $H$ denote the arc $h g$ and $K$ the arc $g k$. By Theorem $3, H^{*}$ and $K^{*}$ are sec plus their interiors. It easily follows that $g \subset \operatorname{Bd}\left(H^{*}\right) \cup \operatorname{Bd}\left(K^{*}\right)$. Since $\operatorname{Bd}\left(H^{*}\right)$ and $\mathrm{Bd}\left(K^{*}\right)$ are scc, it follows that $g$ is a continuous curve, a contradiction. Since $G$ is either a scc or are with respect to its elements, $G$ must be an arc with respect to its elements.

Definition 2. The statement that the compact continuum $C$ in $S$ is circle-like means that $C$ has only two complementary domains and $C$ is the boundary of each.

In the following theorem, $S$ is also assumed to be separable.

THeOREM 7. Let $M$ denote a compact continuum such that (1) $M$ is filled up by a continuous collection $G$ of disjoint continua, (2) all but countably many elements of $G$ are circle-like, and (3) there is a point $0 \notin M$ such that no element of $G$ separates two elements from one another and both from 0 in $S$. Then $G$ is an arc with respect to its elements.

Proof. First let us show that if $g_{1}, g_{2} \in G$, then one of $g_{1}$ and $g_{2}$ separates the other from 0 . Suppose the contrary. Then there exist elements $g_{1}$ and $g_{2}$ of $G$ such that neither separates the other from 0 . Let us now suppose that there is some element $g \in G$ which separates both $g_{1}$ and $g_{2}$ from 0 . Let $H_{1}$ denote a collection such that $X \in H_{1}$ if and only if $X$ separates $g_{1}$ from both 0 and $g_{2}$, or $X=g_{1}$. Let $H_{2}$ denote a collection such that $X \in H_{2}$ if and only if $X=g_{2}$ or $X$ separates $g_{2}$ from both $g_{1}$ and 0 . Let $(A B)$ denote an are such that $(A B) \cap g_{1}=A$ and $(A B) \cap g_{2}=B$. Let $A^{\prime}$ denote the first point in the order from $B$ to $A$ of $(A B) \cap \mathrm{Cl}\left(H_{1}^{*}\right)$, and let $B^{\prime}$ denote the first point 
in the order from $A$ to $B$ of $(A B) \cap \mathrm{Cl}\left(H_{2}^{*}\right)$. Since $M$ is closed, there exist $h_{1}, h_{2} \in G$ such that $A^{\prime} \in h_{1}$ and $B^{\prime} \in h_{2}$. Let $(C D)$ denote an arc such that $g_{1} \cap(C D)=C$ and $g \cap(C D)=D$. Let $D^{\prime}$ denote the first point in the order from $C$ to $D$ of $(C D) \cap \mathrm{Cl}\left(H^{*}\right)$, and let $h \in G$ such that $D^{\prime} \in h$. Now $h_{1} \in H_{1}$. Suppose the contrary, and suppose $h_{1}$ does not separate $g_{1}$ from 0 . Then it follows that $h_{1}$ is a limit element of $H_{1}$. Let $\alpha$ denote an arc such that $0 \in \alpha$ and such that $\alpha \cap g_{1} \neq \varnothing$ but $\alpha \cap h_{1}=\varnothing$. There is a domain $U$ containing $h_{1}$ such that $U \cap\left(g_{1} \cup \alpha\right)=\varnothing$. Since $G$ is a continuous collection and $h_{1}$ is a limit element of $H_{1}$, there is some $t \in H_{1}$ such that $t \subset U$. But since each element of $H_{1}$ separates $g_{1}$ from $0, t \cap \alpha \neq \varnothing$, a contradiction. Similarly, it may be shown that $h_{1}$ separates $g_{1}$ from $g_{2}$. Thus $h_{1} \in H_{1}$. Likewise, it may be shown that $h \in H$ and $h_{2} \in H_{2}$. Now $h_{1} \neq h_{2}$. Otherwise this would imply that $h_{1}$ separates $g_{1}$ from $g_{2}$ and also separates both from 0 , contrary to the hypothesis.

Now $h$ and $h_{1} \cup h_{2}$ are two closed subsets of $G$. Since $G$ is a compact continuum with respect to its elements, there is a subcontinuum $L$ of $G$ which is irreducible from $h$ to $h_{1} \cup h_{2}$. Suppose $t_{1}$ and $t_{2}$ are two circle-like elements of $L-\left(h \cup h_{1} \cup h_{2}\right)$ such that $t_{1}$ separates $t_{2}$ from 0 . Let $L_{1}$ and $L_{2}$ denote subcontinua of $L$ which are irreducible respectively from $h_{1} \cup h_{2}$ to $t_{2}$ and from $h$ to $t_{1}$, and let $E$ denote the complementary domain of $t_{1}$ such that $t_{2} \subset E$. It easily follows that $0 \in E$. Now $t_{2} \subset L_{1}$ or $t_{2} \subset L_{2}$. Suppose $t_{2} \subset L_{1}$. Then $h_{1} \subset E$ or $h_{2} \subset E$, say $h_{1}$. Then it follows that $g_{1} \subset E$ also. This means that $t_{1}$ separates $g_{1}$ from 0 . If $g_{2} \subset E$ also, it follows that $t_{1} \in H_{1}$ contrary to the fact that $h_{1}$ is the outer most element of $H_{1}$. If $g_{2} \subset E$, then $g_{2} \in H_{1}$ again contrary to the fact that $h_{1}$ is the outer most such element. The assumption that $g_{2} \subset E$ leads to the same contradictions. Hence, $t_{2} \notin L_{1}$. Now suppose $t_{2} \in L_{2}$. Then $h \in E$. As before neither $g_{1} \subset E$ nor $g_{2} \subset E$. This means that $h$ does not separate both $g_{1}$ and $g_{2}$ from 0 . Hence, it follows that $t_{2} \in L_{1}$, a contradiction. Thus, no one of the circle like elements of $L-\left(h \cup h_{1} \cup h_{2}\right)$ separates the other from 0 . This means that there exist uncountably many disjoint domains in a separable space, a contradiction.

Let us suppose now that there is no element of $G$ separating both $g_{1}$ and $g_{2}$ from 0 . Let $L$ denote a subcontinuum of $G$ which is irreducible from $h_{1}$ to $h_{2}$, and again suppose there exist two circle-like elements $t_{1}$ and $t_{2}$ of $L-\left(h_{1} \cup h_{2}\right)$ such that $t_{1}$ separates $t_{2}$ from 0 . Again let $L_{1}$ and $L_{2}$ denote two subcontinua of $L$ which are irreducible from $h_{1}$ to $t_{1}$ and from $h_{2}$ to $t_{1}$ respectively. Let $E$ denote the complementary domain of $t_{1}$ such that $t_{2} \subset E$. Now suppose $t_{2} \in L_{1}$. Then it follows $g_{1} \subset E$ which means that $t_{1}$ separates $g_{1}$ from 0 . Now $g_{2} \subset E$ since we are assuming that no element of $G$ separates both $g_{1}$ and $g_{2}$ from 0 . Hence, $t_{1} \in H_{1}$, contrary to the fact that $h_{1}$ is the outer most 
element of $H_{1}$. A similar contradiction is reached when it is assumed that $t_{2} \in L_{2}$. This again means that there are uncountably many disjoint domains. Hence, it must follow that if $g_{1}, g_{2} \in G$, then one must separate the other from 0 . Since $G$ is a compact continuum, $G$ has two noncut "points" $A_{1}$ and $A_{2}$. Now one must separate the other from 0 , say $A_{1}$ separates $A_{2}$ from 0 . Let $A_{3} \in G$ such that $A_{3} \neq A_{1}$ and $A_{3} \neq A_{2}$. Now $A_{3}$ separates $A_{2}$ from 0 or vice versa. Suppose $A_{3}$ separates $A_{1}$ from 0 . Then $A_{1}$ does not separate $A_{3}$ from 0 . But $A_{1}$ does separate $A_{2}$ from 0 . This means that $A_{1}$ is a cut point of $G$, a contradiction. Hence, $A_{3}$ does not separate $A_{1}$ from 0 . Suppose $A_{2}$ separates $A_{3}$ from 0 . Since $A_{2}$ does not separate $A_{1}$ from 0 , we would have $A_{2}$ as a cut point of $G$, a contradiction. Hence $A_{3}$ separates $A_{2}$ from 0 . This means that $A_{3}$ is a cut point of $G$. By definition, $G$ is an arc from $A_{1}$ to $A_{2}$.

Definition 3. We shall say that the compact continuum $M$ in the plane is annulus-like if and only if there exist two circle-like subcontinua $C_{1}$ and $C_{2}$ of $M$ such that if $I_{2}$ is the interior of $C_{2}$ and $I_{1}$ is the interior of $C_{1}$, then $M=C_{1} \cup C_{2} \cup\left(I_{1}-\mathrm{Cl}\left(I_{2}\right)\right)$.

THEOREM 8. Let $M$ denote a compact continuum in the plane which is filled up by a continuous collection of disjoint circle-like continua. Then $M$ is annulus-like.

Proof. By Theorem 7, $G$ is an arc with respect to its elements. Let $C_{i}$ denote the end points of $G$, and let $I_{i}$ denote the interior of $C_{i}, i=1,2$. Let $L=I_{1}-\left(I_{2} \cup C_{2}\right)$, and let $F$ denote a component of $L$. Then $F \cap\left(G-\left(C_{1} \cup C_{2}\right)\right) \neq \varnothing$. Suppose the contrary. Then $\mathrm{Bd}(F) \subset C_{2}$ or $\operatorname{Bd}(F) \subset C_{1}$. For if $\operatorname{Bd}(F) \cap C_{1} \neq \varnothing$ and $\operatorname{Bd}(F) \cap C_{2} \neq \varnothing$, then since each element of $G-\left(C_{1} \cup C_{2}\right)$ separates $C_{1}$ from $C_{2}$, then some element of $G$ must intersect $F$. Suppose $\operatorname{Bd}(F) \subset C_{1}$. Now $I_{1}-F \neq \varnothing$ since $I_{2} \subset I_{1}$, and $I_{1}$ contains a point of $F$ since $F \subset I_{1}$. This means $I_{1} \cap C_{1} \neq \varnothing$, a contradiction. A similar contradiction is reached by assuming $\operatorname{Bd}(F) \subset C_{2}$ using the exterior of $C_{2}$. Now $L$ has only one component since $G-\left(C_{1} \cup C_{2}\right)$ is connected and each component of $L$ intersects some element of $G$. Now suppose $L-M \neq \varnothing$, and let $P \in L-M$. Let $H_{i}$ denote a collection such that $X \in H_{i}$ if and only if $X=C_{i}$ or $X \in G$ which separates $C_{i}$ from $P$, where $i=1,2$. As in Theorem 6 , it follows that there is some outermost element $h_{i}$ in $H_{i}, i=1,2$. Now $h_{1} \neq h_{2}$ since each element is circle-like. Let $h$ denote a point of the interval $h_{1} h_{2}$ of $G$ distinct from both $h_{1}$ and $h_{2}$. It then follows that either $h$ separates $C_{1}$ from $P$ or $h$ separates $C_{2}$ from $P$. In either case a contradiction would be reached since it would mean that $h_{i}$ is not the outermost element in $H_{i}, i=1,2$. 
Hence $M=C_{1} \cup C_{2} \cup L$. Thus $M$ is annulus-like by definition.

\section{REFERENCES}

1. R. D. Anderson, Open mappings of compact continua, Proc. Nat. Acad. Sci. 42 (1956), 247-349.

2. M. E. Hamstrom, Concerning continuous collections of continuous curves, Proc. Amer. Math. Soc. 4 (1953) 240-243.

3. R. L. Moore, Foundations of point set theory, American Mathematical Society Colloquium Publications, Vol. 13, 1962.

Received December 16, 1964.

Clemson University 


\section{PACIFIC JOURNAL OF MATHEMATICS}

H. SAMELSON

Stanford University

Stanford, California

J. P. JaNs

University of Washington

Seattle, Washington 98105

\section{EDITORS}

J. DugundJI

University of Southern California Los Angeles, California 90007

RICHARD ARENS

University of California

Los Angeles, California 90024

\section{ASSOCIATE EDITORS}

E. F. BECKENBACH

B. H. NEUMANN

F. WOLF

K. YosidA

\section{SUPPORTING INSTITUTIONS}

UNIVERSITY OF BRITISH COLUMBIA

CALIFORNIA INSTITUTE OF TECHNOLOGY

UNIVERSITY OF CALIFORNIA

MONTANA STATE UNIVERSITY

UNIVERSITY OF NEVADA

NEW MEXICO STATE UNIVERSITY

OREGON STATE UNIVERSITY

UNIVERSITY OF OREGON

OSAKA UNIVERSITY

UNIVERSITY OF SOUTHERN CALIFORNIA
STANFORD UNIVERSITY

UNIVERSITY OF TOKYO

UNIVERSITY OF UTAH

WASHINGTON STATE UNIVERSITY

UNIVERSITY OF WASHINGTON

AMERICAN MATHEMATICAL SOCIETY CHEVRON RESEARCH CORPORATION TRW SYSTEMS

NAVAL ORDNANCE TEST STATION 


\section{Pacific Journal of Mathematics}

\section{Vol. 20, No. $1 \quad$ September, 1967}

Leonard Daniel Baumert, Extreme copositive quadratic forms. II ........ 1

Edward Lee Bethel, A note on continuous collections of disjoint

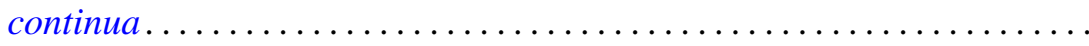

Delmar L. Boyer and Adolf G. Mader, A representation theorem for abelian groups with no elements of infinite p-height ...................

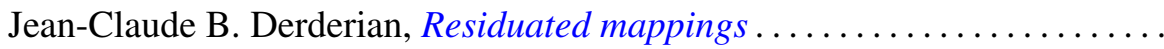

Burton I. Fein, Representations of direct products of finite groups ......... 45

John Brady Garnett, A topological characterization of Gleason parts.......

Herbert Meyer Kamowitz, On operators whose spectrum lies on a circle or

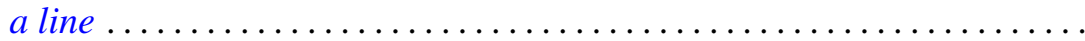

Ignacy I. Kotlarski, On characterizing the gamma and the normal distribution ........................................

Yu-Lee Lee, Topologies with the same class of homeomorphisms ..........

Moshe Mangad, Asymptotic expansions of Fourier transforms and discrete polyharmonic Green's functions ...........................

Jürg Thomas Marti, On integro-differential equations in Banach spaces ....

Walter Philipp, Some metrical theorems in number theory.............. 109

Maxwell Alexander Rosenlicht, Another proof of a theorem on rational

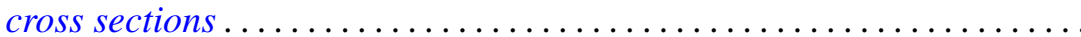

Kenneth Allen Ross and Karl Robert Stromberg, Jessen's theorem on Riemann sums for locally compact groups

Stephen Simons, A theorem on lattice ordered groups, results of Ptak, Namioka and Banach, and a front-ended proof of Lebesgue's theorem...

Morton Lincoln Slater, On the equation $\varphi(x)=\int_{x} x+1 K(\xi) f[\varphi(\xi)] d \xi \ldots$ 155 Arthur William John Stoddart, Existence of optimal controls .. 167 Burnett Roland Toskey, A system of canonical forms for rings on a direct sum of two infinite cyclic groups ....................

Jerry Eugene Vaughan, A modification of Morita's characterization of dimension 